\title{
ACM Springer Mobile Networks and Applications (MONET) Journal Special Issue on Future Internet for Green and Pervasive Media
}

\author{
Jonathan Rodriguez • Rahim Tafazolli • \\ Christos Verikoukis
}

Published online: 11 March 2012

(C) Springer Science+Business Media, LLC 2012

Over the last two decades, mobile services rapidly grew from being an accessory service to becoming an essential part of day to day life, the colloquial saying that "one would never leave home without the house keys" is now the same for the mobile phone. This is reflected in the market penetration of mobile subscriptions over the last decades. Starting in 1991 when the first mobile phone call was made in Finland over the Global System of Mobile Communications (GSM), today we have reached over 4 billion mobile subscriptions, which is more than half of the world population. In comparison, there are only around 1.3 billion fixed line subscribers worldwide. Moreover, according to analytical estimation, the global subscriptions will reach 6 billion in 2014 and is estimated to achieve $100 \%$ global penetration by 2015 , meaning that the number of subscriptions will almost match the world population. With this increase in the number of subscriptions and traffic, the energy and cost of mobile communications will increase as well. Mobile networks are already suffering high energy and financial costs. The energy bill accounts for a large portion of the operational expenditure (OPEX) of mobile telecom providers, and equals $1 \%$ of the monthly subscription income of mobile networks.

\footnotetext{
J. Rodriguez $(\bowtie)$

Instituto de Telecomunicações,

Aveiro, Portugal

e-mail: jonathan@av.it.pt

R. Tafazolli

University of Surrey,

Guildford, UK

e-mail: r.tafazolli@surrey.ac.uk

C. Verikoukis

CTTC,

Barcelona, Spain

e-mail: cveri@cttc.es
}

Energy consumption has now become an important design metric for wireless systems, which before was confined to the familiar energy ratings seen on household appliances. The carbon footprint resulting from mobile phone usage has seen an alarming increase in the last decade with rise in mobile traffic, and the future is not looking rosy. In fact, the whole Information and Communications Technology (ICT) sector has been estimated to account for $2 \%$ of global $\mathrm{CO}_{2}$ emissions, a percentage comparable to the emissions due to global aviation. Mobile networks are estimated to contribute to around 0.2 of global $\mathrm{CO}_{2} \mathrm{e}\left(\mathrm{CO}_{2}\right.$ equivalent $)$ emissions in 2007 and this is expected to increase to $0.4 \%$ in 2020 . While the overall $\mathrm{CO}_{2} \mathrm{e}$ emission of ICT will less than double between 2007 and 2020, the Carbon footprint of mobile networks is predicted to almost triple during the same period, reaching more than one third of current annual emissions of the entire United Kingdom. Both the increase in cost and Carbon footprint of mobile networks mandate urgent solutions to the problem of energy consumption of mobile networks, especially if the EU is to meet the ambitious 20:20:20; a mandate set forth in order to reduce the greenhouse gas emissions and energy consumption by $20 \%$, whilst increasing energy from renewable sources by $20 \%$. This political leverage in unison with the expected forecast for higher mobile traffic demand has placed energy saving at the forefront of system design for telecom operators. Therefore disruptive solutions are needed to solve the problem of increased cost and energy consumption of future mobile networks: if we do not take radical action now then $4 \mathrm{G}$ users will be restricted to the nearest electricity socket providing a hypocritical stance on the $4 \mathrm{G}$ philosophy that preaches unrestricted freedom.

This special issue intends to target some high quality research and practical initiatives in the hot field of green and 
pervasive networking to support future internet applications. The call for papers included topics on disruptive technologies such as cognitive and cooperation for promoting energy saving, but not limited to this. We present here five of the best works that will allow the MONET reader to go beyond stateof-the-art on these topics, and perhaps establish new bridges towards further innovation on this field.

Alonso-Zarate et al. explore cooperation between relays based on Automatic Retransmission Request schemes for energy saving. This notion exploits the broadcast nature of the radio channel by allowing those users which overhear a transmission to act as spontaneous relays when a packet has been received with errors at destination. In the case of error, retransmissions are then executed by the relays, providing the system with cooperative diversity. This paradigm analyzes how the addition of these relays to the communication modifies the well-known hidden and exposed terminal problems associated to any medium access control protocol based on carrier sensing.

The trade-off between energy efficiency and capacity maximization is one of the most challenging issues to be addressed by current and future cellular networks. Kolios et al. investigate this issue by capitalizing on the inherent delay tolerance of Internet type services, and propose a framework where significant energy savings can be achieved by postponing the communication of information for a later time instance when the networking conditions improve. Decentralized store-carry and forwarding schemes were proposed, and in the single-hop case, it was shown that mobile users could achieve 33 times reduction in the communication energy consumption compared to the traditional instantaneous transmission.

N. Costagliola et al. contribute towards the field of energy efficient routing for MANETS. The so called MChannel group communication middleware, a protocol that allows applications to become context-aware of the underlying topology by moving the network protocol to the user space, is optimised to support energy saving and delay efficiency. MChannel makes use of the Optimized Link State Routing (OLSR) protocol and is extended enabling unicast routing based on two alternative metrics, namely end-to-end delay and overall network lifetime. Beyond this, MChannel's approach represents a general framework which can be used by programmers to introduce in MChannel new novel metrics and path selection algorithms.

In addition to the wireless access component, wireless technologies are also gaining popularity for the back-haul infrastructure of cellular systems mainly due to their cost and easy deployment. In this context, Wireless Mesh Networks (WMN) are commonly considered the most suitable architecture because of their versatility to allow flexible configurations. Capone et al. have introduced a synergy between the flexibility of WMN and the need for energy saving by presenting an optimized framework for network management that takes into account the trade-off between the network energy requirements and the daily variations in demand. The simulation results have shown that up to $40 \%$ reduction in energy could be achieved under normal loading conditions.

Our final contribution in this special issue presents an energy efficient (E2) and protocol independent mechanism for Mobile Ad-hoc Networks (MANETs) routing that has potential applications in public safety scenarios. Ramrekha et al. have integrated the E2 mechanism with the scalable ChaMeLeon (CML) protocol resulting in the so called E2CML. Through the integration process of E2-CML, the aim was to satisfy the requirements for emergency MANETs (eMANETs), but beyond that to demonstrate that the proposed mechanism is portable across popular protocols such as Optimized Link State Routing (OLSR) and Ad hoc OnDemand Vector (AODV). Simulation results have shown that the E2CML is scalable, robust and can improve network lifetime over traditional AODV and OLSR routing protocols.

We would like to thank all authors who submitted manuscripts to this special issue and the reviewers that assisted the guest editors to select the best articles for this special issue. Our gratitude also extends to the 2010 edition of the MobiMedia conference, and the C2POWER (248577), CO2GREEN (TEC2010-20823), and GREENET (264759) projects that have provided the launchpad for this special issue.

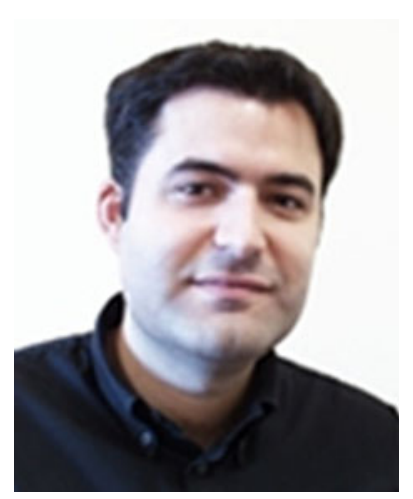

Dr Jonathan Rodriguez received his Masters degree and his $\mathrm{Ph} . \mathrm{D}$ in Electronic and Electrical Engineering from the University of Surrey (UK), in 1998 and 2004 respectively. In 2002, he became a Research Fellow at the Centre for Communication Systems Research and was responsible for coordinating Surrey's involvement in European research projects under Framework Programmes 5 and 6. Since 2005, he has been a Senior Researcher at the Instituto de Telecomunicações (Portugal), and founded the 4TELL Wireless Communication Research Group in 2008. The 4TELL group currently comprises 25 researchers with a project portfolio that includes 10 ongoing European collaborative research projects. He is currently the project coordinator for the Seventh Framework C2POWER project, and technical manager for COGEU. He is author of more than 100 scientific publications, served as general chair for several prestigious conferences and workshops, and has carried out consultancy for major manufacturers participating in DVB-T/H and HS-UPA standardisation. His research interests include green communications, cognitive radio cooperative strategies, radio resource management cross-layer design and baseband digital signal processing. 


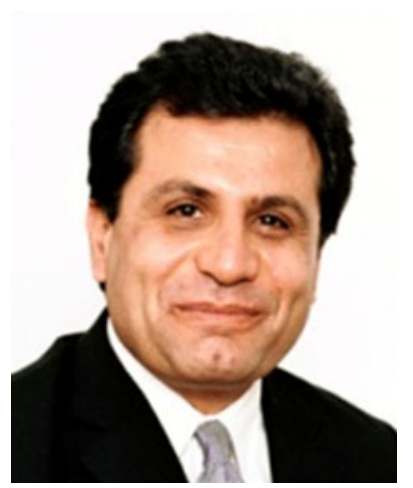

Professor Rahim Tafazolli He is the Director of the Centre for Communications Systems Research (CCSR), Faculty of Engineering and Physical Sciences, The University of Surrey in the UK. He has published more than 500 research papers in refereed journals, international conferences and as invited speaker. He currently has more than 20 patents in the field of mobile communications. He is the editor of two books on "Technologies for Wireless Future" published by Wiley's Vol.1 in 2004 and Vol.2 2006. He is currently chairman of EU Net!Works Technology Platform Expert Group. He is Fellow of IET, WWRF (Wireless World Research Forum) and a Senior member of IEEE.

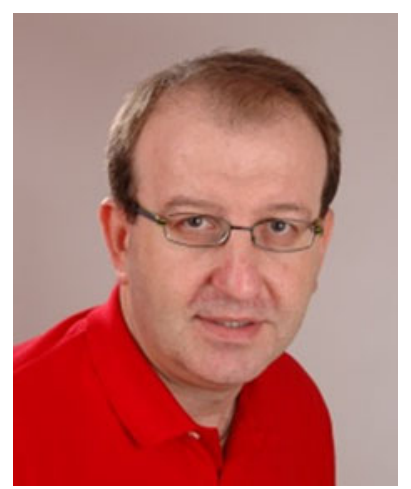

Christos Verikoukis got his $\mathrm{Ph}$. D. from the Technical University of Catalonia in 2000. He is currently a Senior Researcher at CTTC and an adjunct professor at UB. His area of expertise is in the design of energy efficient layer 2 protocols and RRM algorithms, for short range wireless cooperative and network coded communications. Dr. Verikoukis has participated and coordinated several national and European projects. He has published 35 journal papers and over 80 conference papers. He is also co-author in 2 books, 12 chapters in different books and in 2 patents. Dr. Verikoukis has participated more than 20 competitive projects (IST, ICT, CELTIC, MEDEA+, CATRENE, Marie-Curie, COST) while he has served as the Principal Investigator in 3 national projects in Greece and Spain as well as the technical manager in 5 Marie-Curie and 2 Celtic projects. He has served as coeditor in 5 special issues while he has participated in the organization of several international conferences. He is also a regular reviewer in a number of international journals. He has appointed to serve as a reviewer in FP7 projects and as an evaluator in ARTEMIS-JU and for research funded projects in Greece and in Spain. He has supervised 10 Ph.D. students and 2 Post Docs researchers since 2004. He is an IEEE Senior Member. 